\title{
Combining spatial data with survey data improves predictions of boundaries between settlements
}

\begin{abstract}
Studies of land-use change often require the combination of socioeconomic survey data with spatially continuous maps of land-cover change. One approach is to define maps of land ownership, assuming that all land-use change can be attributed to the owners or managers of each parcel of land. Unfortunately, records of administrative boundaries between towns and villages are commonly unavailable in developing countries and prohibitively costly or time consuming to map for individual projects. However, point locations of the settlements themselves can be obtained easily from existing maps or remotely-sensed imagery. In this paper we compare three methods - circular buffers, unweighted Voronoi polygons (sometimes referred to as Thiessen polygons) and multiplicatively weighted Voronoi polygons - for estimating boundaries between villages in an agricultural landscape in West Africa. The benefits and limitations of each approach are discussed, and their accuracy assessed using 98 independently collected GPS coordinates of village boundaries. We present a novel method for generating and optimising weights for multiplicatively weighted Voronoi polygons using survey data of village sizes from a subset of villages. By using both spatial information and survey data from villages, we show that multiplicatively weighted Voronoi polygons outperform other methods of predicting village boundaries, and increase the correlation coefficient between surveyed village area and mapped areas from 0.18 to 0.68 compared with more commonly used unweighted Voronoi polygons. Our method of weighting Voronoi polygons can be implemented with data and software commonly available to researchers and non-governmental organisations.
\end{abstract}

Keywords: Circular buffers, GIS, land-use, Thiessen polygons, Voronoi polygons, West Africa 


\section{Introduction}

The study of human-driven land-cover changes often requires merging spatially continuous, or pixel-level, data of land-cover with spatially discontinuous data from socioeconomic surveys (Liverman \& Cuesta, 2008; Skole, 1994; Walsh \& Welsh, 2003). Household-level or village-level surveys can provide detailed information about people living within a single location. On the other hand, satellite imagery and other remotely sensed products can produce spatially continuous maps of land-cover change through time, over large areas, and often at high spatial resolution. However, understanding the influence of people on the landscape by linking social data with satellite imagery is a key and persistent challenge for land-change science (Liverman and Cuesta, 2008). Whilst some types of survey data, such as population estimates, can be interpolated between points to produce information of population density at the pixel level (Balk, et al., 2006; Herold, et al., 2002), others are aggregated within administrative boundaries to produce panel data, or are categorical in nature such that interpolation of the values is not meaningful (Walsh \& Welsh, 2003). Where the scale of analysis or policy intervention is at the village or settlement level, an understanding of administrative boundaries between settlements is of interest to link measured socioeconomic variables to observed land-cover changes and studying the drivers of land-use change (Irwin \& Geoghegan, 2001; Robinson, et al., 2013). In developed countries, land tenure is often well-established and administrative boundaries between farms or towns are generally accurately mapped and publicly available. In developing countries, however, the data on private land holdings and fine-scale administrative boundaries, such as between towns and villages, are commonly unmapped (Blackman, 2013). Even where land tenure is wellunderstood locally it can be prohibitively costly or infeasible to map these administrative boundaries for large study areas (Liverman, et al., 1998). But, unlike administrative boundaries that might have no physical basis, the locations of the settlements themselves are 
often available even for remote regions, since they can be mapped quickly using hand-held GPS units or remotely with high resolution aerial imagery from satellites or aircraft (Herold, et al., 2002; Lu, et al., 2008). In the absence of better records, these point-locations can be used to predict the administrative boundaries between settlements.

Looking beyond land change science to other disciplines studying spatial processes, two approaches for dividing landscapes between point data have been favoured to date. The first and simplest uses only data about each point to generate a circular buffer around them, ignoring the spatial arrangement and properties of other points in the landscape (Pigot, et al., 2010). The size of the buffer is determined from assumptions about the influence of the generator point (in the case of settlements for example, villages might be assumed to manage land in a radius that is proportional to their population). This approach has been used in studies across numerous academic fields, including to examine access to retailers (Duncan, et al., 2014), in neutral models of species ranges (Baselga, et al., 2012; Pigot, et al., 2010) and species occupancy models (Efford \& Dawson, 2012). The benefits of circular buffers are in their simplicity; they require only the location of each point and some information about range. As new data are collected, they may be easily added to the study landscape, as each point is treated as independent of the others. However, a strong drawback of circular buffers is that they result in ranges that may overlap, and they have uniform shapes. Whilst this may be an acceptable for some spatial phenomenon, it is typically an unrealistic result when mapping land ownership.

The second approach, “Thiessen” or unweighted Voronoi polygons, does not account for differences in the influence of each generator point and instead uses only their spatial configuration to divide the landscape through tessellations (Okabe, et al., 2008a). A tessellation is the complete division of a defined portion of Cartesian plane into non- 
overlapping regions given a finite number of generator points (Okabe, et al., 2008b). Unweighted Voronoi polygons are constructed such that each polygon contains only one generator point, and any area within a polygon is closer to its associated point than any other. They therefore assume that, for any given location, the most likely land manager is from in the nearest village (measured by Euclidean distance to the village settlement). Unlike circular buffers, they require no information about the points other than their spatial configuration. Unweighted Voronoi polygons have been used to map bird territories (Schlicht, et al., 2014), determine movement of organisms across landscapes (Galpern \& Manseau, 2013), map vegetation (Gooding, et al., 1997), create neighbourhoods for housing market studies (Kryvobokov, 2013) and for land-use models (Muller \& Zeller, 2002). However, unweighted Voronoi polygons assume that all generator points have the same influence in the landscape. This may not be the case if, for example, settlements with larger populations are expected to have more land than those with small populations, regardless of their location. Unweighted Voronoi polygons also require a complete dataset of all villages within the bounds of the study area; any missing villages will lead to an overestimation in the size of neighbouring polygons and introduce error to the results.

This paper presents a third approach, weighted Voronoi polygons, and compares its effectiveness against circular buffers and unweighted Voronoi polygons as a method for estimating village boundaries in a region of West Africa. Weighted Voronoi polygons combine both the spatial configuration of generator points with additional information about the expected size or influence of each point. Starting with an unweighted Voronoi tessellation, polygon sizes are adjusted by multiplying the distance measures between points by a weighting term for each point (Okabe, et al., 2008b). Multiplicatively weighted Voronoi polygons have been used in some research fields including in ecology to predict species growth and competition (Aakala, et al., 2013; Du, et al., 2012), generate theoretical forest 
landscapes (Li, et al., 2010; Passolt, et al., 2013) and study territory sizes and shapes (Adams, 2001; Wilkin, et al., 2006). They have also been used in studies of urban hierarchies in the US (Mu \& Wang, 2006), in logistics (Galvão, et al., 2006) and in measuring consumer preferences (Boots \& South, 1997). However, to our knowledge there are no other studies that use multiplicatively weighted Voronoi polygons to determine administrative boundaries between settlements. Here we present a novel method for generating the village-level weightings using incomplete village-level survey data. Methods for generating the weighted Voronoi polygons were adapted from Dong (2008).

\section{Methods}

\subsection{Study area}

Our study area is 286,200 hectares of community land outside of the Gola Rainforest National Park (GRNP) in Sierra Leone, West Africa (Figure 1). The landscape is managed predominantly for subsistence slash-and-burn agriculture and, with the exception of isolated private estates and the National Park itself, land is almost exclusively owned and managed by 476 villages within the seven chiefdoms around the National Park. Despite the region recovering from a civil war ending in 2002, administrative boundaries between villages are well-defined by local institutions. However, the demarcations have not been officially mapped or registered with the government. For this study we assume that village lands are contiguous and contain the village that owns them, and that all land is owned by only one village with no shared land. Using local knowledge, we believe these assumptions to be reasonable for our study area, however the methods should be adapted for areas where these assumptions do not hold. 


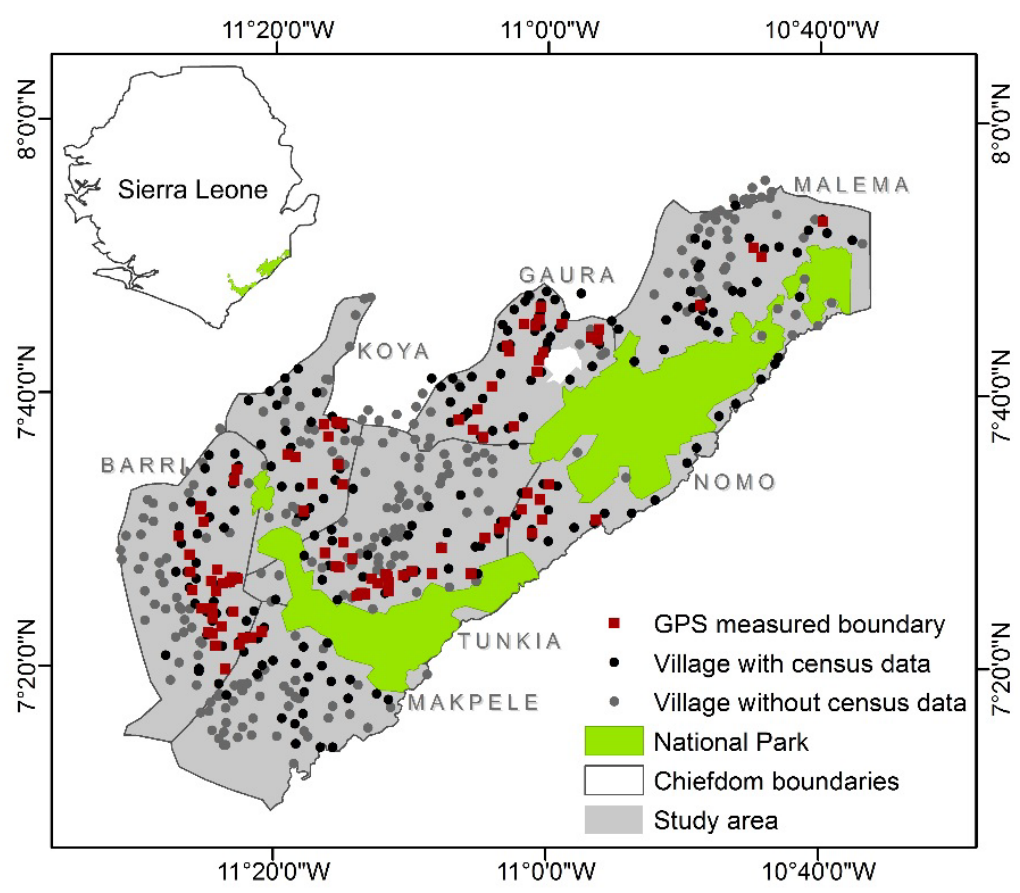

Figure 1. Study site in Sierra Leone, West Africa. Villages surround the Gola Rainforest National Park, across seven chiefdoms. The distribution of GPS coordinates at known boundaries between villages are shown.

The location of all 476 inhabited villages is known a priori from an existing dataset of coordinates provided by the Gola National Park Program and Royal Society for the Protection of Birds (RSPB), updated and adjusted using high resolution RapidEye satellite imagery collected in 2010. In 2010 and 2011, 228 of the villages were visited by researchers from the University of Wageningen, and socioeconomic census data were collected using village-level surveys. The survey included a question asking for an estimate of the total village area, including all land belonging to the village, farmed or otherwise. For 98 of the 228 surveyed villages, the survey teams also used handheld GPS devices to record the coordinates of the boundary between one village and the next as they crossed on foot, verified by local guides. This produced 98 point recordings of the boundaries between villages, and these can be used as an independent dataset against which the performance of different methods can be tested. On average, most surveyed villages will have two recorded boundary 
locations, one where the teams entered onto the land belonging to the village, and one where they exited. However, in regions where neighbouring villages were also surveyed, some villages may have more than two recordings of boundary locations.

\subsection{Circular neighbourhood buffers}

Responses from the 2010-2011 surveys of estimated village area are used to generate circular village boundaries around 228 villages using the buffer function in ArcGIS 10.0. The circles have a radius (r) such that the area of the generated circle is the same as the total village area reported in the surveys, and $r=\sqrt{ }$ Area $/ \pi$. The 248 villages that did not have survey data were excluded from this part of the analysis.

\subsection{Unweighted Voronoi polygons}

The landscape is divided using Thiessen polygons function in ArcGIS 10.0, generated from the point shapefile of all 476 village coordinates. Thiessen polygons are equivalent to unweighted Voronoi polygons. The polygons are calculated from a Delaunay triangulation, whereby vectors are created between the points to form triangles such that the minimum angle of all the angles in the triangulation is maximised. The perpendicular bisectors of these triangles form the edges of the unweighted Voronoi polygons (see Dong 2008 for more information). Known commercial or large-scale land holdings and the national park are masked from the study area using the clip function in ArcGIS 10.0.

\subsection{Weighted Voronoi polygons}

Weighted Voronoi polygons are created following the raster-based methodology described by Dong (2008) using the 'RPyGeo' package v.0.9-3 in R to run python geoprocessing scripts from ArcGIS 10.0. Each village acts as a generator point, and for each point a matrix of Euclidean distances is created for the study extent at $30 \mathrm{~m} \times 30 \mathrm{~m}$ resolution. The output raster 
is computed as the minimum value of all 476 distance rasters, on a cell by cell basis. This output raster could be interpreted as an elevation raster, with each generator point being a valley in the landscape and midway between generator-points having the highest values and representing ridges or peaks (Figure 2). To convert this elevation raster to polygons the flow direction function in the ArcGIS 10.0 hydrology module is used to compute the difference in elevation between each cell and its neighbouring cells. Second, the basin tool is applied to delineate drainage basins from the flow direction raster by identifying regions at the edge of the study area where water would run off the surface (pour points), and calculating the area that would contribute to each pour point. This raster is then converted to a polygon of village lands. Without weighting, these polygons are equivalent to unweighted Voronoi polygons.

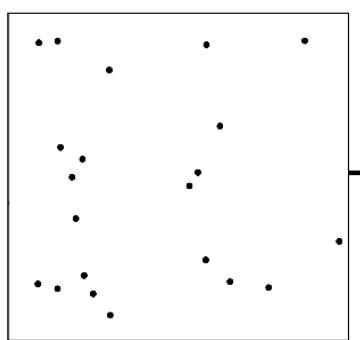

Village shapefile

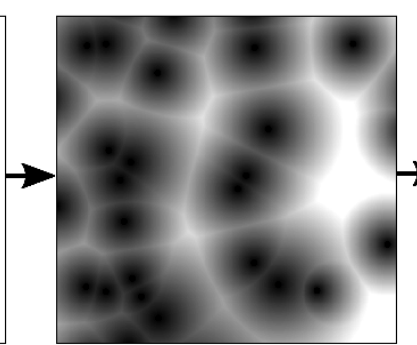

Elevation raster

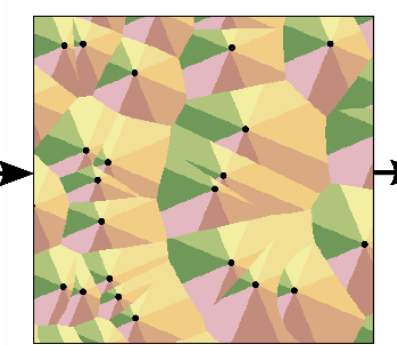

Flow direction raster

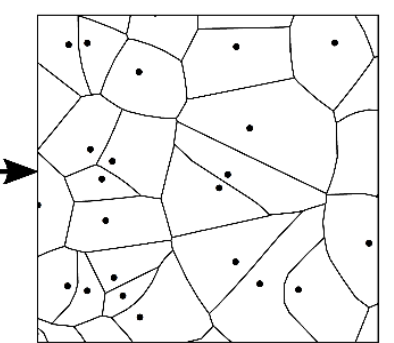

Basin shapefile

Figure 2. Construction of multiplicatively-weighted Voronoi polygons using raster-based method and hydrology modules from ArcGIS. Method adapted from Dong (2008).

To weight the polygons by village characteristics, the Euclidean distance raster for each village is multiplied by a weighting for that village, prior to the output raster being computed. A weight between 0 and 1 will increase the influence of that village, while a weight $>1$ will reduce its relative influence. Villages with a surveyed area greater than that predicted by unweighted Voronoi polygons are assigned a weight less than one, and those where the reported area was less than the corresponding unweighted Voronoi polygon are given a weight greater than one. Villages with the same area as predicted by unweighted Voronoi polygons (a difference of zero), are assigned a weighting of one and their distance matrices 
are therefore unchanged. A logistic function is used to create weights for the polygons, with upper (upr) and lower (lwr) bounds of 1.9 and 0.1 respectively, and one free parameter $\sigma$ determining the slope of the function (Equation 1). This function has the properties of being symmetrical around the point $(0,1)$ to ensure that villages with a predicted area equal that reported in the surveys are assigned a weighting of one. The upper and lower bounds are chosen as limits to the weighting function to constrain the weighting from being dominated by extreme values in the survey data. For small values of sigma, the function does not reach the asymptotic values so this is only used for larger values of sigma. These limits can be adjusted for each study, and should be large enough to allow the minimum of the cost function to be found (see below).

$$
\text { weights }=u p r+\frac{(l w r-u p r)}{1+e^{(\sigma * d i f f)}}
$$

\section{Equation 1}

Where diff is the difference between the surveyed size of the village and the area predicted by unweighted Voronoi polygons. Smaller values of $\sigma$ reduce the range of weights assigned to the villages, and a sigma value of zero is equivalent to unweighted Voronoi polygons as it will assign all villages with a weight of 1 (Figure 3).

A cost function is calculated as the difference between the village area predicted by weighted Voronoi polygons and the reported village area from survey data, averaged across 223 villages. This cost function is calculated for a range of sigma values $>0$, and a quadratic function fitted to the cost function values with respect to sigma. The minimum of this function is the value of sigma where the predicted village areas most closely match those reported in the census survey, on average, and this is the value of sigma that is therefore used to create the final weighted Voronoi polygons. 

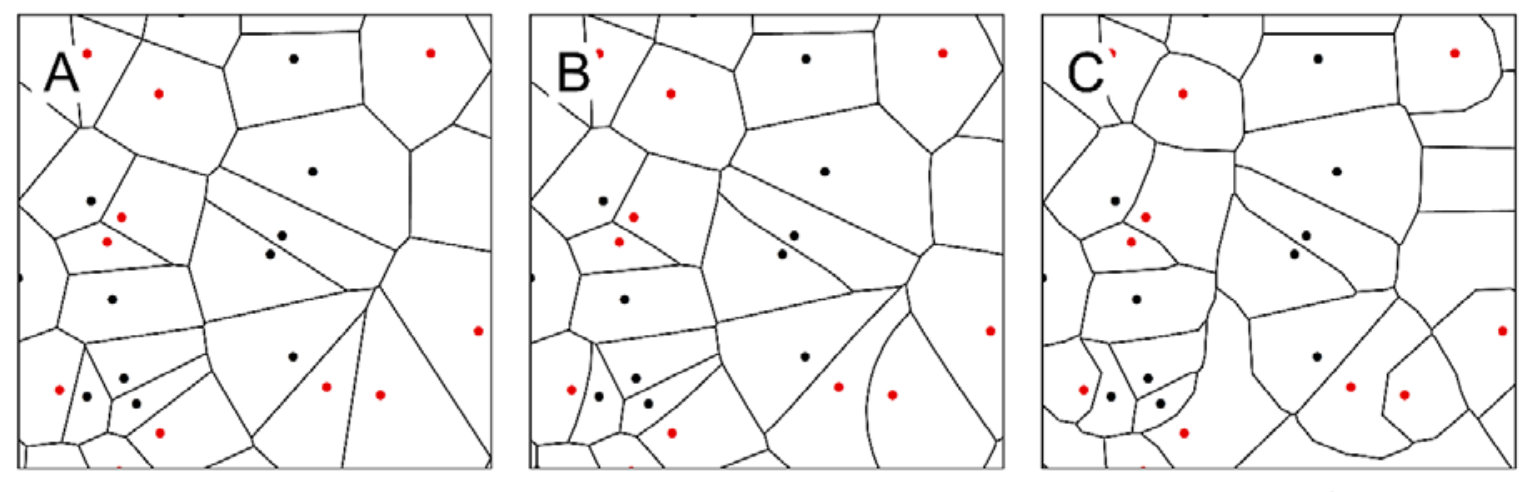

Increasing weighting parameter, $\sigma$

Figure 3. Effect of changing the weighting parameter, $\sigma$, in multiplicatively-weighted Voronoi polygon generation. Panel (A) $\sigma$ of zero and is therefore equivalent to an unweighted Voronoi polygon, panel (B) and panel (C) have increasing values of $\sigma$. Red points indicate villages with survey data, black points are villages without survey data and therefore assigned a weight of one.

\subsection{Assessing model performance}

The performance of the three approaches is assessed against a dataset of coordinates for 98 known village boundaries, collected by survey teams in the field. The deviation between predicted and measured village boundaries is calculated as the mean absolute Euclidean distance between these known points and the closest polygon edge predicted by the different methods. Polygons are converted to polyline files in ArcGIS 10.0, before using the near function to calculate distances. To account for the multiple overlapping circular buffers, the circles are layered by their ranked area such that smaller buffers are above larger buffers. Using the dissolve function, the shapes are then aggregated together based on their size and thereafter converted to a polyline file. 
For unweighted Voronoi and multiplicatively-weighted Voronoi polygons, the areas of the final polygons per village are also compared with reported village sizes for the 228 surveyed villages using correlation coefficients, and described using the $\mathrm{Im}$ function in R. Circular buffers are not compared as their area is equal to the reported village size, by design.

\section{Results}

\subsection{Circular neighbourhood}

Boundaries predicted by circular buffers have substantial overlap between neighbouring village extents (Figure 4 A). Model performance is not assessed against reported village areas as there would be a perfect correlation, however the predicted model boundaries are compared against 98 known boundary locations has a mean distance of $360.7 \mathrm{~m}$ per village.
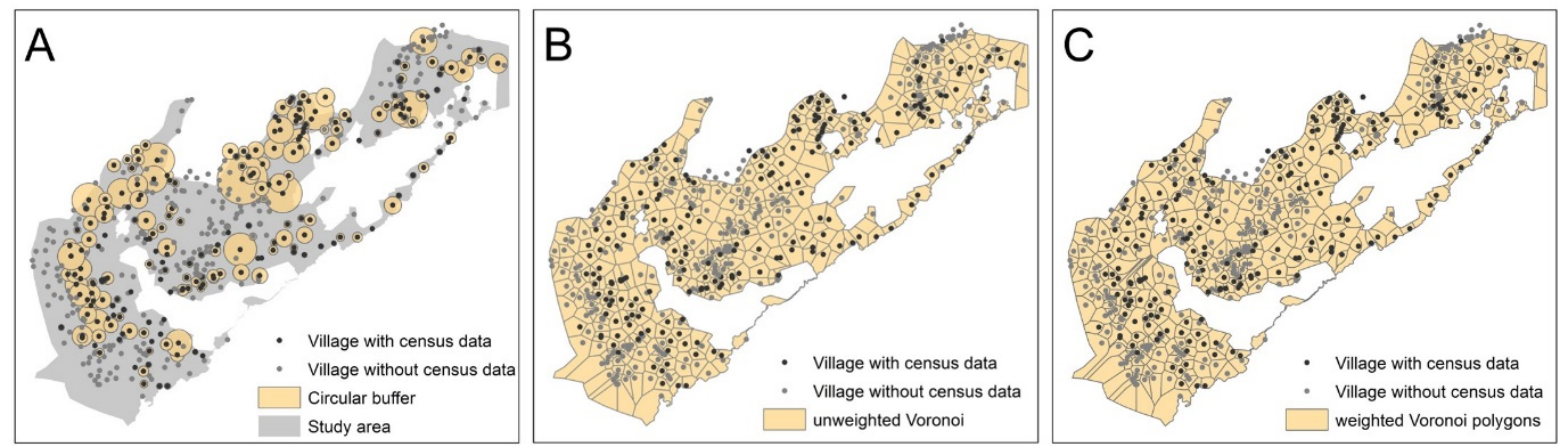

Figure 4. Three alternative methods for dividing and aggregating land-cover data by village (A) buffers of known area around each village; (B) Unweighted Voronoi polygons; (C) Multiplicativelyweighted Voronoi polygons. The study area is defined as the boundary of the seven chiefdoms associated with the National Park.

\subsection{Unweighted Voronoi polygons}


When village areas generated by unweighted Voronoi polygons are compared to the village area reported in surveys, the relationship is very weak (correlation coefficient 0.18 ; Figure 5 A). Despite a few cases where the unweighted Voronoi polygons substantially underestimate the size of a village's land compared with the census data, in most cases the unweighted Voronoi polygons are larger than the reported village extent, shown by a regression gradient of less than one. For very small villages $\left(<4 \mathrm{~km}^{2}\right.$, where the 1:1 line and regression line intersect; Figure 5 A), the unweighted Voronoi polygons underestimate village size as reported in the surveys, so the spatial distribution of villages does not appear to be sufficient to predict their land holdings. The boundaries predicted by unweighted Voronoi polygons deviate from measured boundaries by a mean of $318.6 \mathrm{~m}$ per village (Table 1 ).
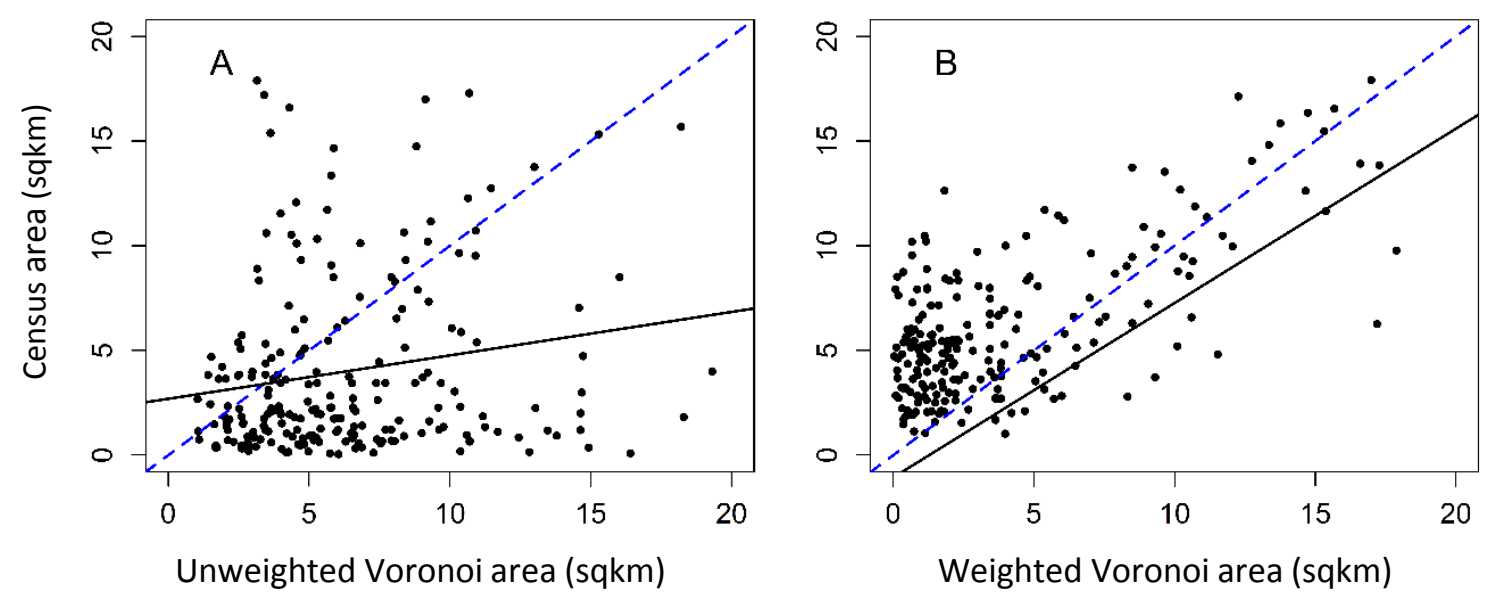

Figure 5. Relationship between area of village land estimated from census data and (A) area calculated by unweighted Voronoi polygons, or (B) area predicted by final weighted Voronoi polygons. The blue dashed line shows the expected 1:1 relationship between the variables; the black solid line is a linear regression between the variables. Unweighted Voronoi polygons have a correlation coefficient of 0.18 with census area, compared with a correlation coefficient of 0.68 for weighted Voronoi polygons. The line of best fit in panel A is described by the function $0.83 x-1.06$, in panel $B$ the line of best fit is described by the function $0.21 x+2.69$. 


\subsection{Multiplicatively-weighted Voronoi polygons}

The value of $\sigma$ that minimised the cost function was found to be 0.000375 (Figure 6). The deviation of boundaries predicted by weighted Voronoi polygons from measured GPS points improved compared with unweighted Voronoi polygons by 7\%, and compared with circular buffers by 18\% (mean = $295.8 \mathrm{~m}$; Table 1 ). The correlation coefficient between predicted village area and surveyed village area is improved substantially in the weighted Voronoi polygons, from 0.18 for unweighted Voronoi polygons to 0.68 for the weighted Voronoi polygons (Figure $4 \mathrm{~B}$ ). The gradient of the regression line $(0.83)$ indicates that the relationship between weighted Voronoi polygons and surveyed areas is much closer to the desired 1:1 relationship compared with unweighted Voronoi polygons. Although the regression line for weighted Voronoi polygons is still above the 1:1 line, indicating that predicted areas are larger than the surveyed areas in general, this difference is now more consistent across village sizes (Figure $4 \mathrm{~B}$ ). In addition to improving the mean and median of the cost function, weighted Voronoi polygons also reduce the variability of the cost function (Table 1), indicating that the prediction of village boundaries is more precise using weighted compared with unweighted Voronoi polygons.
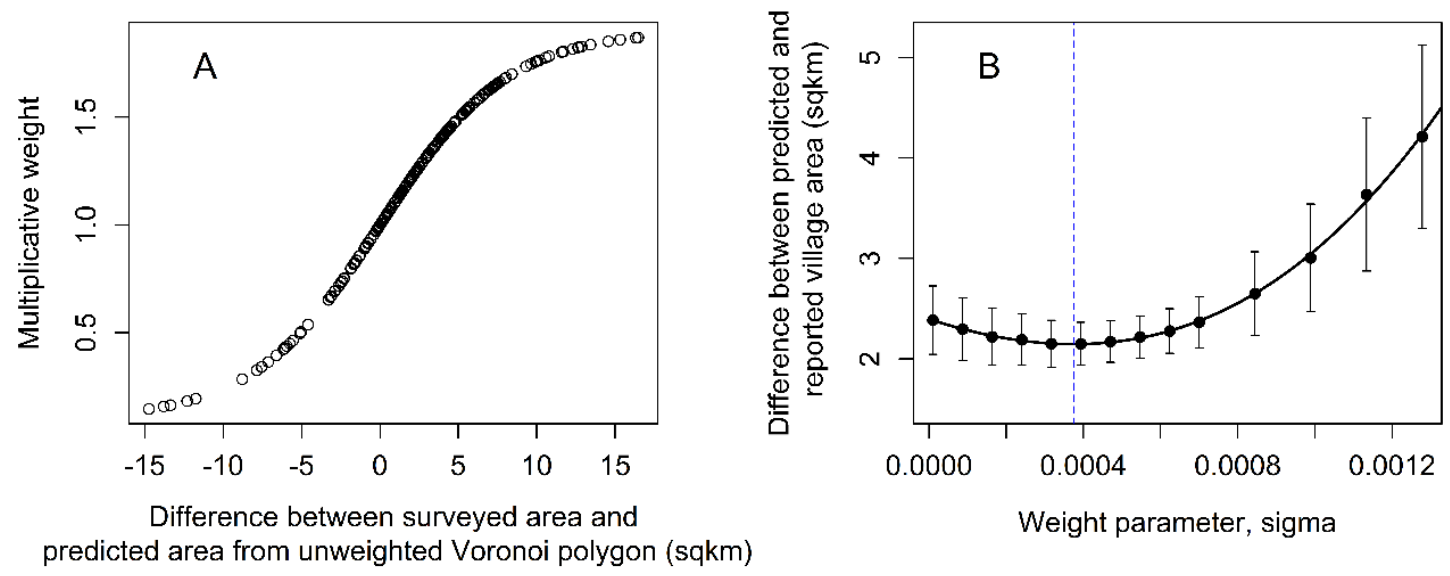
Figure 6. (A) Example of function describing multiplicative Voronoi weights with respect to difference in village area between unweighted Voronoi prediction and that reported from surveys, for $\sigma=0.001$. (B) Cost function with respect to a range sigma values. The cost function is calculated as the predicted village area from Voronoi polygon minus the reported village area from survey data, averaged across 223 villages. The quadratic function fitted to the data is $(6.23 \mathrm{E}+08) \sigma 3+$ $(1.30 \mathrm{E}+06) \sigma 2+(-1.24 \mathrm{E}+03) \sigma+2.40$ with respect to the weight parameter $\sigma$. The blue dashed line indicates the minimum of this function, and therefore the best value of $\sigma$.

Table 1. Summary of deviations of predicted vs measured village boundaries, calculated as the distance between 98 measured coordinates of village boundaries and the closest boundary predicted by each method.

\begin{tabular}{llll}
\hline Approach & $\begin{array}{l}\text { Mean distance } \\
(\mathrm{m})\end{array}$ & $\begin{array}{l}\text { Median distance } \\
(\mathrm{m})\end{array}$ & $\begin{array}{l}\text { Inter-quartile } \\
\text { range }(\mathrm{m})\end{array}$ \\
\hline Circular buffer & 360.7 & 257.1 & 482.3 \\
Unweighted Voronoi & 318.6 & 206.8 & 394.1 \\
Weighted Voronoi & 295.8 & 195.3 & 365.4 \\
\hline
\end{tabular}

\section{Discussion}

This paper has presented three approaches to predicting property boundaries between settlements, and found that weighted Voronoi polygons performed better than either unweighted Voronoi polygons or circular buffers when tested against an independent dataset.

Circular buffers were the worst performing method of the three that we assessed. Unlike the unweighted and weighted Voronoi methods, this approach cannot infer village boundary 
information from the spatial arrangement of villages and therefore excludes any villages without survey data (over 200 in our study area). The buffer approach also assumes that the village settlement lies centrally within the village lands, which is unlikely to be true given the irregular arrangement of villages in the landscape. A downside of the buffer approach for this study is the prediction of significant regions of overlap between neighbouring villages, often covering multiple settlements within one village buffer. If this approach were used to infer land ownership, observed land-cover changes could not be reliably assigned to one village, making this method inadequate for many land-cover change studies (Agarwal, et al., 2002; Irwin \& Geoghegan, 2001). Whilst the strength of the circular buffer approach is in its simplicity and suitability for incomplete datasets, it does require village-specific data order to set the neighbourhood radius. The accuracy of the result is dependent, in part, on the quality of the information used to set the radius value. It is known that estimation of distances and areas elicited in survey data can be inaccurate (McBride, et al., 2012) and relying on these data alone to set the village boundaries, combined with an assumption of circular village extents, has been shown here to yield poor results.

Unweighted Voronoi polygons divide the study area completely between the villages, and follow the intuitive assumption that land-cover change is most likely caused by people living in the nearest village to the location of change. However, one result of such a triangulation is that the spatial distribution of the villages alone will determine the area of the polygons. Villages clustered together will be assigned small land areas due to the limited distance between them and the next village, while remote villages are assigned large areas of land. We have shown that this over and under-estimation leads to a larger variance in the errors of predicted property boundaries, and a lower accuracy compared with weighted Voronoi polygons that include additional information about village sizes. 
Weighted Voronoi polygons combine the benefits of village-specific information used in circular buffers with information inferred from the spatial configuration of villages used in unweighted Voronoi polygons, and perform better than either as a result. Whilst estimates of village areas elicited in surveys can have high uncertainty (Mc Bride et al. 2012), they have been shown here to be valuable for improving estimates of village boundaries compared with methods that ignore survey data and rely purely on spatial approaches to estimate village boundaries. The improvement in predictive accuracy by weighting polygons by surveyed village sizes is modest (7\%), but the use of these imperfect data to generate village weightings allowed a large number of villages in the study area to be weighted, due to the high coverage of surveyed villages. Of course, were a larger number of GPS points available, these data could have been split and a subset of GPS points used as training data to create village weightings instead of the survey data. Such an approach using more accurate training data could be expected to produce further improvements in village boundary predictions. Like unweighted Voronoi polygons, weighted Voronoi polygons of any kind also require a complete dataset of village locations and are vulnerable to overestimating village areas if the locations of some villages are missing from the dataset.

The method presented here allows Voronoi weightings to be set using typically available village-level survey data, and both optimising and updating these weights using easily collectible field data. The weighted Voronoi polygons were created using open source $\mathrm{R}$ software and commonly available ArcGIS software used by many land-use and conservation practitioners. The raster-based approach employed in this study is more computationally intensive than vector-based approaches used to create unweighted Voronoi polygons in ArcGIS 10.0, and can therefore be time consuming to compute at very high spatial resolutions or for large datasets (Dong, 2008). 
Understanding who is the owner or manager of a parcel of land is an important step in merging socioeconomic survey data with spatially continuous land-cover in models of landuse change (Liverman \& Cuesta, 2008; Skole, 1994; Walsh \& Welsh, 2003). Inaccurate assumptions about land tenure introduce error into models (Robinson, et al., 2013), and the method presented here provides more accurate estimates of land boundaries than two other commonly used methods. More broadly, as collaboration between socioeconomic sciences and natural sciences continues to expand, methods are needed to effectively combine data of multiple types to maximise our understanding of land-use systems, particularly in data-poor regions of the world. Even where the datasets are incomplete or of low quality, we have shown that our most accurate model is achieved when multiple datasets can be included. 


\section{Acknowledgements}

We are grateful to Dr Maarten Voors and other researchers from the University of Wageningen for sharing survey data and GPS locations collected from the study site in Sierra Leone. Funding for this research was contributed by the Cambridge Conservation Initiative and the Natural Environment Research Council (NE/H52449x/1). These funding bodies had no involvement in the study design, analysis, interpretation of the data, or writing of the report. We are not aware of any conflict of interest. 


\section{References}

Aakala, T., Fraver, S., D'Amato, A. W., \& Palik, B. J. (2013). Influence of competition and age on tree growth in structurally complex old-growth forests in northern Minnesota, USA. Forest Ecology and Management, 308, 128-135.

Adams, E. S. (2001). Approaches to the study of territory size and shape. Annual Review of Ecology and Systematics, 32, 277-303.

Agarwal, C., Green, G. M., Grove, J. M., Evans, T. P., \& Schweik, C. M. (2002). A review and assessment of land-use change models: Dynamics of space, time, and human choice. In: Center for the study of institutions, population, and environmental change, Indiana University.

Balk, D. L., Deichmann, U., Yetman, G., Pozzi, F., Hay, S. I., \& Nelson, A. (2006). Determining global population distribution: methods, applications and data. In A. G. Simon I. Hay \& J. R. David (Eds.), Advances in Parasitology (Vol. Volume 62, pp. 119-156): Academic Press.

Baselga, A., Lobo, J. M., Svenning, J.-C., \& Araújo, M. B. (2012). Global patterns in the shape of species geographical ranges reveal range determinants. Journal of Biogeography, 39, 760-771.

Blackman, A. (2013). Evaluating forest conservation policies in developing countries using remote sensing data: An introduction and practical guide. Forest Policy and Economics, 34, 1-16.

Boots, B., \& South, R. (1997). Modeling retail trade areas using higher-order, multiplicatively weighted voronoi diagrams. Journal of Retailing, 73, 519-536.

Dong, P. (2008). Generating and updating multiplicatively weighted Voronoi diagrams for point, line and polygon features in GIS. Computers \& Geosciences, 34, 411-421. 
Du, F., Xu, X., Zhang, X. C., Sui, Y., Shao, M., Hu, L., \& Shan, L. (2012). The relationships between aboveground biomass and Voronoi area of coexisting species in an old-field community. Polish Journal of Ecology, 60, 479-489.

Duncan, D. T., Kawachi, I., Subramanian, S. V., Aldstadt, J., Melly, S. J., \& Williams, D. R. (2014). Examination of how neighborhood definition influences measurements of youths' access to tobacco retailers: a methodological note on spatial misclassification. American Journal of Epidemiology, 179, 373-381.

Efford, M. G., \& Dawson, D. K. (2012). Occupancy in continuous habitat. Ecosphere, 3, 32.

Galpern, P., \& Manseau, M. (2013). Finding the functional grain: Comparing methods for scaling resistance surfaces. Landscape Ecology, 28, 1269-1281.

Galvão, L. C., Novaes, A. G. N., Souza De Cursi, J. E., \& Souza, J. C. (2006). A

multiplicatively-weighted Voronoi diagram approach to logistics districting. Computers and Operations Research, 33, 93-114.

Gooding, R. F., Rackham, D., Holland, J. P., \& Robertson, D. (1997). Detailed surveying and mapping of plant communities on featureless terrain. Grass and Forage Science, 52, 439-444.

Herold, M., Scepan, J., \& Clarke, K. C. (2002). The use of remote sensing and landscape metrics to describe structures and changes in urban land uses. Environment and Planning A, 34, 1443-1458.

Irwin, E. G., \& Geoghegan, J. (2001). Theory, data, methods: Developing spatially explicit economic models of land use change. Agriculture, Ecosystems and Environment, 85, 7-23.

Kryvobokov, M. (2013). Hedonic price model: defining neighbourhoods with Thiessen polygons. International Journal of Housing Markets and Analysis, 6, 79-97. 
Li, R., Bettinger, P., \& Weiskittel, A. (2010). Comparisons of three different methods used to generate forest landscapes for spatial harvest scheduling problems with adjacency restrictions. Mathematical and Computational Forestry and Natural-Resource Sciences, 2, 53-60.

Liverman, D., \& Cuesta, R. M. R. (2008). Human interactions with the Earth system: people and pixels revisited. Earth Surface Processes and Landforms, 33, 1458-1471.

Liverman, D., Moran, E. F., Rindfuss, R. R., \& Stern, P. C. (1998). People and pixels: linking remote sensing and social science. Washington, D.C.: National Academy Press.

Lu, D., Tian, H., Zhou, G., \& Ge, H. (2008). Regional mapping of human settlements in southeastern China with multisensor remotely sensed data. Remote Sensing of Environment, 112, 3668-3679.

McBride, M. F., Garnett, S. T., Szabo, J. K., Burbidge, A. H., Butchart, S. H. M., Christidis, L., Dutson, G., Ford, H. A., Loyn, R. H., Watson, D. M., \& Burgman, M. A. (2012). Structured elicitation of expert judgments for threatened species assessment: a case study on a continental scale using email. Methods in Ecology and Evolution, 3, 906920.

Mu, L., \& Wang, X. (2006). Population landscape: A geometric approach to studying spatial patterns of the US urban hierarchy. International Journal of Geographical Information Science, 20, 649-667.

Muller, D., \& Zeller, M. (2002). Land use dynamics in the central highlands of Vietnam: a spatial model combining village survey data with satellite imagery interpretation. Agricultural Economics, 27, 333-354.

Okabe, A., Boots, B., Sugihara, K., Chiu, S. N., \& Kendall, D. G. (2008a). Introduction. In Spatial Tessellations (pp. 1-42): John Wiley \& Sons, Inc. 
Okabe, A., Satoh, T., Furuta, T., Suzuki, A., \& Okano, K. (2008b). Generalized network Voronoi diagrams: Concepts, computational methods, and applications. International Journal of Geographical Information Science, 22, 965-994.

Passolt, G., Fix, M. J., \& Tóth, S. F. (2013). A Voronoi tessellation based approach to generate hypothetical forest landscapes. Canadian Journal of Forest Research, 43, 78-89.

Pigot, A. L., Owens, I. P. F., \& Orme, C. D. L. (2010). The environmental limits to geographic range expansion in birds. Ecology Letters, 13, 705-715.

Robinson, B. E., Holland, M. B., \& Naughton-Treves, L. (2013). Does secure land tenure save forests? A meta-analysis of the relationship between land tenure and tropical deforestation. Global Environmental Change (in press).

Schlicht, L., Valcu, M., \& Kempenaers, B. (2014). Thiessen polygons as a model for animal territory estimation. Ibis, 156, 215-219.

Skole, D. L. (1994). Data on global land-cover change: acquisition, assessment, and analysis: Cambridge University Press.

Walsh, S. J., \& Welsh, W. F. (2003). Approaches for linking people, place, and environment for human dimensions research. GeoCarto International, 18, 51-61.

Wilkin, T. A., Garant, D., Gosler, A. G., \& Sheldon, B. C. (2006). Density effects on lifehistory traits in a wild population of the great tit Parus major: Analyses of long-term data with GIS techniques. Journal of Animal Ecology, 75, 604-615. 